\title{
Changes in Brain Electrical Activity According to Post-traumatic Stress Symptoms in Survivors of the Sewol Ferry Disaster: A 1-year Longitudinal Study
}

\author{
Sehee Jin ${ }^{1}$, Cheolmin Shin ${ }^{1}$, Changsu Han ${ }^{1}$, Yong-Ku Kim ${ }^{1}$, Jongha Lee ${ }^{1}$, Sang Won Jeon ${ }^{2}$, Seung-Hoon Lee ${ }^{3}$, \\ Young-Hoon Ko ${ }^{1}$ \\ ${ }^{1}$ Department of Psychiatry, Korea University College of Medicine, ${ }^{2}$ Department of Psychiatry, Kangbuk Samsung Hospital, Sungkyunkwan \\ University School of Medicine, ${ }^{3}$ Department of Psychiatry, Veterans Health Service Medical Center, Seoul, Korea
}

\begin{abstract}
Objective: The pathology of post-traumatic stress disorder (PTSD) is associated with changes in brain structure and function, especially in the amygdala, medial prefrontal cortex, hippocampus, and insula. Survivors of tragic accidents often experience psychological stress and develop post-traumatic stress symptoms (PTSS), regardless of the diagnosis of PTSD. This study aimed to evaluate electroencephalographic changes according to PTSS in victims of a single traumatic event.

Methods: This study enrolled 60 survivors of the Sewol ferry disaster that occurred in 2014 from Danwon High School and collected electroencephalographic data through 19 channels twice for each person in 2014 and 2015 (mean 451.88 [standard deviation 25.77] days of follow-up). PTSS was assessed using the PTSD Checklist-Civilian Version (PCL-C) and the participants were divided into two groups according to the differences in PCL-C scores between 2014 and 2015. Electroencephalographic data were converted to three-dimensional data to perform low-resolution electrical tomographic analysis.

Results: Significant electroencephalographic changes over time were observed. The group of participants with worsened PCL-C score showed an increased change of delta slow waves in Brodmann areas 13 and 44, with the largest difference in the insula region, compared to those with improved PCL-C scores.

Conclusion: Our findings suggests that the electrophysiological changes in the insula are associated with PTSS changes.
\end{abstract}

KEY WORDS: Post-traumatic stress disorder; Post-traumatic stress symptoms; Electroencephalography; Insula.

\section{INTRODUCTION}

Post-traumatic stress disorder (PTSD) is defined as an exposure to significant traumatic events resulting in four clustered symptoms observed for at least 1 month after the event (intrusions, avoidance of trauma-related thoughts and activities, negative alteration in cognition and mood, and changes in arousal) based on the Diagnostic and Statistical Manual of Mental Disorders, fifth edition (DSM-5)

Received: October 15, 2020 / Revised: November 3, 2020 Accepted: November 4, 2020

Address for correspondence: Young-Hoon Ko

Department of Psychiatry, Korea University Ansan Hospital, Korea University College of Medicine,123 Jeokgeum-ro, Danwon-gu, Ansan 15355, Korea

E-mail: koyh@korea.ac.kr

ORCID: https://orcid.org/0000-0002-5352-2158 criteria [1]. PTSD is a clinically important psychiatric disorder, with a lifetime prevalence of approximately $8 \%$ in the general population of United State and $2 \%$ of South Korea, and imposes a large psychological burden [2-4].

In South Korea, on April 16, 2014, a tragic accident named the Sewol ferry disaster occurred. The ferry, carrying 475 passengers, sank at the southwestern tip of Korea, killing more than 300 people. Of the 325 high school students on board, 250 drowned, leaving only 75 survivors. The surviving students experienced fears of death from a human disaster and sadness after losing close friends. Previous studies have reported an increased incidence of post-traumatic stress symptoms (PTSS) in adolescents who have experienced a difficult disaster, which they were unable to overcome [5-7].

About $70 \%$ of people experience at least one traumatic

(ㄷ) This is an Open-Access article distributed under the terms of the Creative Commons Attribution Non-Commercial License (http://creativecommons.org/licenses/by-nc/4.0) which permits unrestricted non-commercial use, distribution, and reproduction in any medium, provided the original work is properly cited. 
event in their lifetime [8]. Although exposure to traumatic events may increase the risk of PTSD, only fractions of trauma-exposed individuals develop PTSD [9]. Previous studies have identified biological changes in people with PTSS and have shown that the pathology of PTSD is associated with changes in brain structure and function, especially in the amygdala, medial prefrontal cortex, hippocampus, anterior cingulate cortex, and insula [10-12]. However, most of these studies have limitations in that they were cross-sectional studies, compared the patient group to the non-exposed control group, or targeted individuals who had experienced various types of traumatic events. Therefore, there is a need to identify objective changes through longitudinal studies in a homogeneous group that had experienced a single kind of trauma.

Electroencephalography (EEG) is often employed to measure electrical brain signals because of its advantages such as easy handling, non-invasiveness, excellent temporal resolution, and localization of signals within the brain [13]. However, EEG is known to have the disadvantage of having poor spatial resolution in generally, which makes it difficult to infer the location of the brain regions that generate neuronal activity measured on the scalp. In the standardized low-resolution brain electromagnetic tomography (sLORETA) software, EEG of the scalp is calculated and reconstructed three-dimensionally [14]. sLORETA have the benefit of superior time resolution of EEG measurements of milliseconds, and also have spatial resolution of approximately $7 \mathrm{~mm}$, which is similar to that of functional magnetic resonance imaging (fMRI) $[15,16]$. Because SLORETA is a tool that provides highly accurate localization, it is effective at identifying which cortical areas show changes in activity.

The existing EEG studies including SLORETA studies did not show absolute or consistent results in patients with post-traumatic stress symptoms. A quantitative EEG study showed low theta power in developed PTSD group compared to resilient group, but the other study showed increased theta power on central brain regions and beta activity in frontal, central, and occipital regions $[17,18]$. A study comparing the resting EEG of PTSD patient with control group with no history of the trauma showed no significant difference on any of the spectral bands between two groups [19]. Lower activity of the low theta band $(4-5 \mathrm{~Hz})$ was observed in the right temporal lobe and in both frontal lobes in the PTSD patient group com- pared to the control group in a sLORETA study [20]. The other sLORETA study showed a widespread increase of theta activity $(4.5-7.5 \mathrm{~Hz})$ in parietal lobes and in frontal lobes in PTSD patients compared to the control group. The present study aimed to determine which biophysiologic changes were associated with PTSS and localize them through longitudinal observations in a group of participants who had experienced the same trauma.

\section{METHODS}

\section{Participants}

This study enrolled survivors of the Sewol ferry disaster who agreed to participate in the psychiatric approach. All participants were same-grade students of Danwon High School. They had been hospitalized at Korea University Ansan Hospital to receive care for psychological trauma in April 2014, where self-reported questionnaires, such as PTSD Checklist-Civilian Version (PCL-C), Patient Health Questionnaire-9 (PHQ-9), Brief Resilience Scale (BRS), Athens Insomnia Scale (AIS), were administered and initial EEG recordings were obtained. From July to August 2015, 1 year after the initial evaluation, we followed up the participants to evaluate changes in psychological stress through the same questionnaires and EEG measurements. The average of the follow-up duration is 451.88 (standard deviation [SD] 25.77) days. This study was performed retrospectively using the results of clinical scales and EEG recordings, which are routine psychiatric examinations of our hospital. Fourteen subjects were excluded for refusal to respond to the self-reported questionnaires and undergo EEG measurements; thus, a total of 60 medical records were included in the analyses. The research protocol was approved by the Institutional Review Board of Korea University Ansan Hospital for research involving human subjects (No. 2014AS0290).

\section{EEG Recordings and Data Analysis}

EEG was performed for 10 minutes under an eyes-closed resting state in a comfortable and quiet room. The participants were kept awake and were not disturbed in a sitting position. EEG was performed using the Neuronics 32 system (Intermed Co., Ltd., Seoul, Korea) with $19 \mathrm{Ag} / \mathrm{AgCl}$ scalp electrodes arranged on a head cap according to the International 10-20 system [21]. The amplifier bandwidths varied from 1 to $50 \mathrm{~Hz}$, with a $60 \mathrm{~Hz}$ notch filter, 
and the EEGs were digitized at $200 \mathrm{~Hz}$ per epoch. The impedances at all electrodes were maintained below $10 \mathrm{k} \Omega$ during the measurements.

Artifact-free epochs were selected with $>90 \%$ split-half reliability and $>90 \%$ test-retest reliability, with a total epoch duration of 60 seconds. The statistical properties of the segments were calculated using NeuroGuide software (https://appliedneuroscience.com). Artifacts were eliminated using the artifact rejection toolbox in the NeuroGuide software and visual inspection. The selected EEG data were quantified using fast Fourier transform and split into seven frequency bands: delta $(1.5-6.0 \mathrm{~Hz})$, theta $(6.5-$ $8.0 \mathrm{~Hz})$, alpha1 $(8.5-10.0 \mathrm{~Hz})$, alpha2 $(10.5-12.0 \mathrm{~Hz})$, beta1 $(12.5-18.0 \mathrm{~Hz})$, beta2 $(18.5-21.0 \mathrm{~Hz})$, and beta3 $(21.5-30.0 \mathrm{~Hz})$.

For source localization, the EEG data were converted to three-dimensional data and analyzed using standardized low-resolution brain electromagnetic tomography (sLORETA), which is a tomographic method to map EEG data from sensor space to cortical source (also known as EEG source modeling) and is based on images of standardized current density $[14,16,22]$. Independent $t$ tests was performed to compare baseline (2014) EEG power of each band between PLC-C and improved PLC-C score groups based on the threshold for statistical significance $(p<0.05)$. Independent $t$ test was also conducted to compare the changes of sLORETA current densities from 2014 to 2025 between the two groups. The nonparametric permutation was used to correct for multiple comparisons tests performed for each picture contrast between the two groups [23].

\section{PTSD Checklist-Civilian Version (PCL-C)}

PTSS was assessed using the PTSD Checklist (PCL). Among the three versions of the PCL (military, civilian, and specific), we used the civilian version. This self-reported rating scale for PTSD comprises 17 items reflecting the DSM-IV symptoms of PTSD. The participants indicated their frequencies of experiencing PTSD symptoms in the past month on a 5-level scale from "not at all" (1) to "extremely" (5). The sum of the scores served as the total PTSS severity score.

An ideal cutoff for the PCL-C score has not been reported. Depending on the prevalence and setting characteristics, a wide range of PCL-C scores have been proposed as cutoffs for PTSD screening or to aid the diag- nostic assessment of PTSD. The present study focused on the changes in PCL-C scores between 2014 and 2015 rather than a cutoff score or the diagnosis of PTSD. In real-world practice, $80 \%$ of individuals who are exposed to extreme trauma do not develop PTSD [24]. Hence, it is more common for psychiatrists to care for patients with PTS who do not meet the criteria for PTSD. Thus, we classified the participants into two groups according to the difference in PCL-C scores between 2014 and 2015 to determine the changes in EEG recordings according to changes in PTSS: worsened and improved PCL-C score groups. The worsened group comprised 34 survivors with the same score or a positive difference in PCL-C score in 2015 compared to that in 2014, whereas the improved group comprised 26 patients with a negative difference in PCL-C scores between 2015 and 2014.

\section{Patient Health Questionnaire-9 (PHQ-9)}

The PHQ-9 is a self-administered scale derived from the full $\mathrm{PHQ}$, which is a 3-page questionnaire. The PHQ-9 comprises nine items and is designed to measure the severity of depression. It focuses exclusively on the nine diagnostic criteria for DSM-IV depressive disorder. Each item is rated from 0 (not at all) to 3 (nearly every day). Thus, the total score ranges from 0 to 27 [25].

\section{Brief Resilience Scale (BRS)}

The BRS is a simple scale consisting of six questions assessing the ability to "bounce back" or recover from stress. Three questions (Questions 1, 3, and 5) are positively worded, while three (Questions 2, 4, and 6) are negatively worded. One to five points are given for each question and scores for the negative questions are reversed and summed. The higher the total score, the higher the resilience [26].

\section{Athens Insomnia Scale (AIS)}

The AIS is a self-report questionnaire used to measure the intensity of sleep difficulties. The AIS comprises eight questions: five assess nocturnal sleep problems and three estimate the next-day consequences of insomnia. Each item is scored from 0 (no problem at all) to 3 (very serious problem), with the total score ranging from 0 to 24 . Higher AIS scores indicate the presence of clinically significant insomnia symptoms. 
Table 1. Clinical characteristics of the participants

\begin{tabular}{|c|c|c|c|}
\hline Variable & Improved PCL-C score group & Worsened PCL-C score group & $p$ value \\
\hline \multicolumn{4}{|l|}{ Sex } \\
\hline Male & 16 & 11 & $0.024^{*}$ \\
\hline Female & 10 & 23 & \\
\hline Age (yr) & $16.12 \pm 0.33$ & $16.29 \pm 0.46$ & 0.099 \\
\hline \multicolumn{4}{|l|}{ Scale scores in 2014} \\
\hline PCL-C & $35.42 \pm 13.62$ & $25.09 \pm 9.33$ & $0.001 *$ \\
\hline PHQ-9 & $5.23 \pm 5.94$ & $2.85 \pm 3.51$ & 0.077 \\
\hline AIS & $5.62 \pm 4.62$ & $3.24 \pm 3.21$ & $0.022 *$ \\
\hline BRPS & $19.58 \pm 5.78$ & $19.65 \pm 4.87$ & 0.960 \\
\hline IQ & $101.54 \pm 10.82$ & $103.15 \pm 12.44$ & 0.602 \\
\hline \multicolumn{4}{|l|}{ Scale scores in 2015} \\
\hline PCL-C & $26.69 \pm 12.41$ & $35.53 \pm 13.01$ & $0.010^{*}$ \\
\hline PHQ-9 & $4.50 \pm 5.13$ & $6.50 \pm 5.45$ & 0.154 \\
\hline AIS & $5.69 \pm 4.02$ & $5.44 \pm 3.78$ & 0.805 \\
\hline \multicolumn{4}{|c|}{ Changes in clinical scale scores ${ }^{a}$} \\
\hline$\triangle$ PLC-C & $-8.73 \pm 9.04$ & $10.44 \pm 9.82$ & - \\
\hline$\Delta \mathrm{PHQ}-9$ & $-0.73 \pm 2.78$ & $3.65 \pm 4.28$ & $<0.001 *$ \\
\hline$\Delta \mathrm{AIS}$ & $0.23 \pm 3.45$ & $2.21 \pm 3.26$ & $0.027^{*}$ \\
\hline
\end{tabular}

Values are presented as number only or mean \pm standard deviation.

PCL-C, post-traumatic stress disorder checklist-civilian version; PHQ-9, patient health questionnaire-9; AIS, athens insomnia scale; BRPS, brief psychiatric rating scale; $\mathrm{IQ}$, intelligence quotient.

${ }^{a}$ Scale scores in 2015 - scale scores in 2014.

${ }^{*} p<0.05$.

\section{Statistical Analyses}

All data in this study are expressed as arithmetic means \pm SDs, where appropriate. Descriptive analyses were performed to evaluate the baseline characteristics of the participants. We used chi-square and Student's $t$ tests for categorical and continuous variables, respectively. Data analyses and descriptive statistics were performed using IBM SPSS Statistics for Windows, version 22.0 (IBM Corp., Armonk, NY, USA). The results were considered statistically significant if the $p$ value was $<0.05$ (two-tailed).

\section{RESULTS}

\section{Clinical Characteristics}

Although the data of 74 participants were collected initially, 14 participants were excluded due to the absence of completed questionnaires and EEG results. Finally, this study enrolled 60 patients. The clinical characteristics of the participants are provided in Table 1. Of the participants, $45.9 \%$ were male and the mean age of the participants was $16.2 \pm 0.4$ years. The mean PCL-C scores in 2014 and 2015 were $29.36 \pm 12.41$ and $31.95 \pm 13.32$, respectively. There was no significant difference in age, intelligence quotient, or BRS scores between the groups.
There were more male participants in the improved PCL-C score group and more female participants in the worsened PCL-C score group.

The changes in the clinical scale scores after 1 year are also provided in Table 1. There was a significant difference in the changes in PHQ-9 scores over a 1-year period between the worsened and improved PCL-C score groups $(p<0.001)$. The mean change in AIS score also was significantly different between the two groups $(p=0.027)$.

\section{EEG Recordings}

We observed no significant differences between the worsened and improved PCL-C score groups for any band of baseline activity in 2014 ( $p=0.821)$.

However, significantly different changes of activity in the delta band were observed between 2014 and 2015 in the worsened PCL-C score group compared to those in the improved PCL-C score group (Fig. 1). The group with worsened PCL-C score showed a increased change in delta power, and the region with the largest difference was localized to Brodmann area 13 of the left sublobar insular gyrus $(X=-35, Y=-5, Z=20, t=4.15, p=0.017)$. Increased change of delta activity was additionally observed in Brodmann area 44 of the left precentral gyrus $(X=$ 


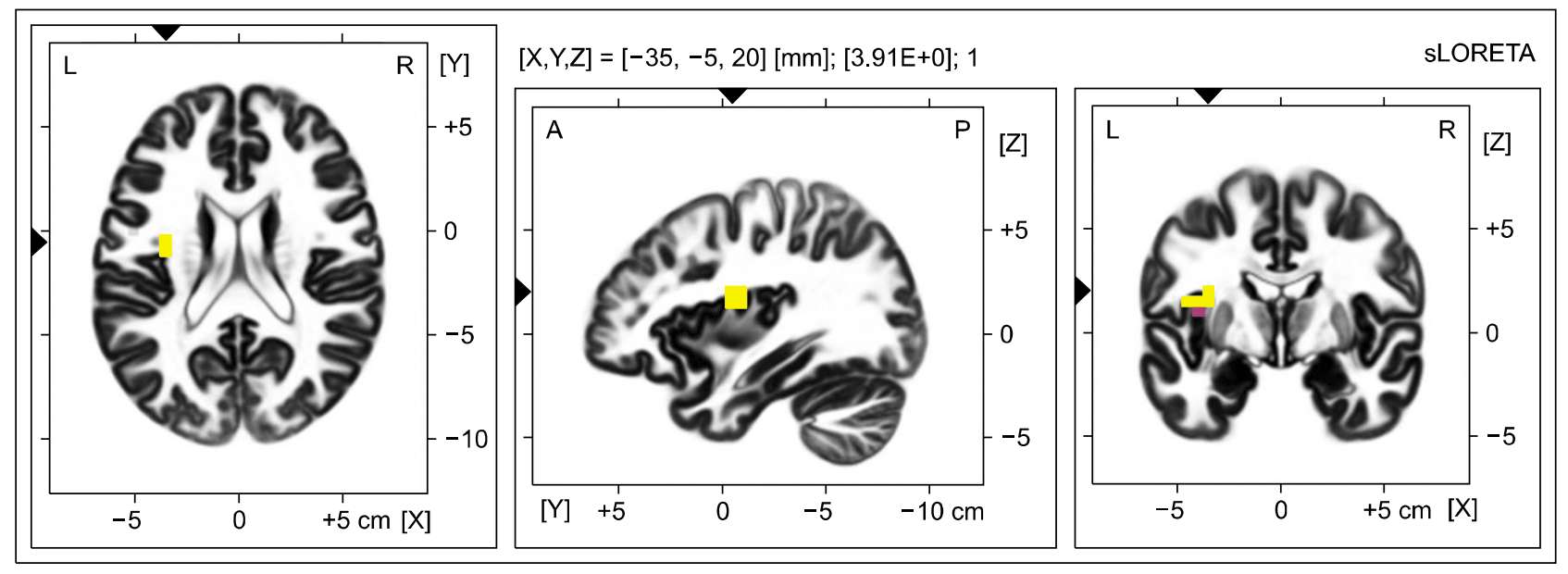

Fig. 1. A significant increase in the delta power spectra was detected on electroencephalography between 2014 and 2015 in the worsened group compared to that in the improved group. The red and yellow colors indicate increased cortical activity. The maximal difference was found in the left sublobar insular gyrus. The statistical significance was set at $p<0.05$.

$-45, \mathrm{Y}=0, \mathrm{Z}=10, \mathrm{t}=3.89, p<0.05)$. The threshold for significance was $t=3.801$, corresponding to a $p$ value of $<0.05$. We did not observe significant differences of EEG changes in the other frequency bands.

\section{DISCUSSION}

In this study, sLORETA revealed a significant difference between the groups with worsened and improved PCL-C scores, with higher changes of delta power in the left sublobar insular gyrus and prefrontal gyrus in the group with worsened scores. Increased changes of delta activity in insula means relatively enhanced slow wave activity in insula, indicating altered functioning, possibly left insular dysfunction, in PTSS psychopathology.

The insula is a triangular area of the brain located beneath the Sylvian fissure. It is covered by the opercula of the frontal, parietal, and temporal lobes $[27,28]$. Neurons in the insular cortex exhibit broad and dense interconnections; thus, the insula is involved in emotion; attention; and verbal, motor, visual, olfactory, gustatory, and somatosensory data processing $[29,30]$. The role of the insula in psychiatric disorders, including PTSD, has received much attention $[28,31]$.

A previous voxel-based analysis using magnetoencephalograms showed focally enhanced slow wave $(1-4$ $\mathrm{Hz}$ ) activity in the insula region of the PTSD group compared to that in the control group [32]. Furthermore, in several neuroimaging studies, PTSD patients showed a re- duction in the gray matter volume of the insula compared to that in controls [33-35]. Structural brain magnetic resonance imaging (MRI) studies of combat-exposed US military veterans showed smaller volumes of the left insula, subgenual anterior cingulate cortex, caudate, and hypothalamus in the PTSD group compared to those in the trauma-exposed healthy control group [36]. The regional cerebral metabolic rate of glucose in the (right) anterior insula and adjacent prefrontal and striatal areas was lower in the PTSD group than that in the recovery group in an 18fluoro-2-deoxyglucose positron emission tomography (FDG-PET) study of trauma-exposed individuals [37]. Based on these results, the study suggested that glucose metabolism in the anterior insula might be related to resilience after trauma and vulnerability to PTSD development. A long-term FDG-PET study on PTSD (20 years after trauma experience) reported relatively diminished activity in the insular region [38].

Altered functional connectivity between the insula and dorsal anterior cingulate cortex or amygdala was observed in PTSD patients [38,39]. The insula is engaged in anticipation processes and fear conditioning, and studies have suggested that changes in insular activity may be related to the hyperarousal symptoms of PTSD. The anterior insula is a key area involved in interoceptive awareness, integrating emotionally potent stimuli with body arousal, and providing a representation of subjective feeling states [40].

The anterior insula has been suggested to interfere with the integration of fragmented somatic sensation and emo- 
tional states into declarative memories, which may lead to dissociative phenomena [41]. A fMRI study using scriptdriven imagery responses showed a negative correlation between dissociative symptoms in the PTSD group and anterior insula activation [42]. Decreased functional connectivity of the vestibular nuclei with the parieto-insular vestibular cortex was observed in dissociative subtype PTSD in resting-state $\mathrm{fMRI}$ studies, suggesting that dysregulation of this integration contributes to dissociative symptoms [43].

Emotional face processing in PTSD patients showed that childhood maltreatment was negatively correlated with amygdala-insular connectivity and activation of the insula and dorsal anterior cingulate cortex during the processing of fear and anger [44]. Individuals who recovered from PTSD after prolonged exposure therapy showed increased functional connectivity between the insular and cingulate regions and changes in functional activation, especially in the left anterior insula, in an fMRI study [45]. In a resting-state $\mathrm{fMRI}$ study comparing PTSD patients and individuals who had experienced trauma without PTSD using the amplitude of low-frequency fluctuation (ALFF) to reflect the intrinsic functional baseline activity of the brain, PTSD patients showed decreased ALFF in the insula, right lingual gyrus, cuneus, middle occipital gyrus, and cerebellum [46].

Delta power is generally associated with decreased sleep and alertness [47]. However, it is also related to the situation in which the person is awake but the body is at rest. It is known that there is a strong correlation between power of delta wave and the default mode network $(\mathrm{DMN})$ in previous studies. The parahippocampal gyrus and the delta power within the DMN showed highly significant correlation in a simultaneous fMRI-EEG study [48]. Less delta power was observed in individuals experiencing greater psychological distress in adults who had experienced depression [49]. A greater change in the delta waves, generated in the insula in the worsened PTSS group than in the improved PTSS group in this study, may mean that the worsened PTSS group needs more DMN activity for recovery. This is contrary to the previous results in which a decrease in DMN activity was observed in a study of PTSD patients [50]. Since this study was not conducted in clinical PTSD patients, results may differ from those in PTSD patients. However, this is consistent with the result of increased functional connectivity of DMN in depressed patients with ruminations [51].
We speculate that people experiencing acute trauma who do not meet the criteria for PTSD may still experience psychological stress and require medical intervention. However, most previous studies have focused on patients with confirmed PTSD. Thus, more attention to symptoms and changes irrespective of diagnosis is needed in clinical practice settings. By identifying differences in objective measurements such as EEG between the PTSS groups with improved and worsened scale scores, we may proactively prevent symptom aggravation.

Our study has several limitations in terms of the generalizability of the findings. The first is the comorbidity of depression. Because of the common comorbidity of depression in patients who suffer from PTSD [52-54], the findings of this study may have resulted from neurophysiological changes in depression. Second, since this study employed a retrospective design, selection bias was possible. To generalize our results, a prospective study is needed. Third, we classified the participants into two groups according to the change of PCL-C scores which reflect PTSS between 2014 and 2015, however, did not consider the cut off value dividing the severity of symptoms.

Despite these limitations, our study had the unique strength of homogeneity of study participants. The population of this study, who had the same educational experience of the same grade, controlled for the diversity of traumatic events and population differences, which may be confounding factors in PTSD studies. Based on electrophysiological data obtained immediately and 1 year after the accident, we could speculate the knowledge of longitudinal neurophysiological changes. In this study, we observed an enhanced change of delta wave in the insula region in the group with worsened PTSS scale scores. This finding suggests that the electrophysiological changes in the insula are associated with PTSS changes.

\section{- Conflicts of Interest}

No potential conflict of interest relevant to this article was reported.

\section{Author Contributions}

Conceptualization: Young-Hoon Ko, Changsu Han. Data acquisition: Sehee Jin, Sang Won Jeon, Seung-Hoon Lee, Cheolmin Shin. Formal analysis: Sehee Jin, Cheolmin Shin. Supervision: Young-Hoon Ko, Yong-Ku Kim, Jongha Lee. Writing-original draft: Sehee Jin, Cheolmin Shin. 
Writing - review \& editing: Cheolmin Shin, Jongha Lee, Young-Hoon Ko. All authors have read and approved the final manuscript.

\section{ORCID}

Sehee Jin

Cheolmin Shin

Changsu Han

Yong-Ku Kim

Jongha Lee

Sang Won Jeon

Seung-Hoon Lee

Young-Hoon Ko

https://orcid.org/0000-0001-9136-4982

https://orcid.org/0000-0002-8232-2921

https://orcid.org/0000-0002-4021-8907

https://orcid.org/0000-0001-5694-7840

https://orcid.org/0000-0003-0824-8564

https://orcid.org/0000-0002-7828-3296

https://orcid.org/0000-0001-5341-0933

https://orcid.org/0000-0002-5352-2158

\section{REFERENCES}

1. American Psychiatric Association. Diagnostic and statistical manual of mental disorders: DSM-5. Washington, D.C.:American Psychiatric Publishing;2013.

2. Kilpatrick DG, Resnick HS, Milanak ME, Miller MW, Keyes KM, Friedman MJ. National estimates of exposure to traumatic events and PTSD prevalence using DSM-IV and DSM-5 criteria. J Trauma Stress 2013;26:537-547.

3. Jeon HJ, Suh T, Lee HJ, Hahm BJ, Lee JY, Cho SJ, et al. Partial versus full PTSD in the Korean community: prevalence, duration, correlates, comorbidity, and dysfunctions. Depress Anxiety 2007; 24:577-585.

4. Cho MJ, Chang SM, Hahm BJ, Chung IW, Bae A, Lee YM, et al. Lifetime risk and age of onset distributions of psychiatric disorders: analysis of national sample survey in South Korea. Soc Psychiatry Psychiatr Epidemiol 2012;47:671-681.

5. Goenjian AK, Walling D, Steinberg AM, Karayan I, Najarian LM, Pynoos R. A prospective study of posttraumatic stress and depressive reactions among treated and untreated adolescents 5 years after a catastrophic disaster. Am J Psychiatry 2005; 162:2302-2308.

6. Roussos A, Goenjian AK, Steinberg AM, Sotiropoulou C, Kakaki M, Kabakos C, et al. Posttraumatic stress and depressive reactions among children and adolescents after the 1999 earthquake in Ano Liosia, Greece. Am J Psychiatry 2005; 162: 530-537.

7. Goenjian AK, Karayan I, Pynoos RS, Minassian D, Najarian LM, Steinberg AM, et al. Outcome of psychotherapy among early adolescents after trauma. Am J Psychiatry 1997;154. 536-542.

8. Benjet C, Bromet E, Karam EG, Kessler RC, McLaughlin KA, Ruscio AM, et al. The epidemiology of traumatic event exposure worldwide: results from the World Mental Health Survey Consortium. Psychol Med 2016;46:327-343.

9. Koenen KC, Ratanatharathorn A, Ng L, McLaughlin KA, Bromet EJ, Stein DJ, et al. Posttraumatic stress disorder in the World Mental Health Surveys. Psychol Med 2017:47:2260-
2274.

10. Bremner JD. Functional neuroimaging in post-traumatic stress disorder. Expert Rev Neurother 2007;7:393-405.

11. Hughes KC, Shin LM. Functional neuroimaging studies of post-traumatic stress disorder. Expert Rev Neurother 2011; 11:275-285.

12. Karl A, Schaefer M, Malta LS, Dörfel D, Rohleder N, Werner A. A meta-analysis of structural brain abnormalities in PTSD. Neurosci Biobehav Rev 2006;30:1004-1031.

13. Butt M, Espinal E, Aupperle RL, Nikulina V, Stewart JL. The electrical aftermath: brain signals of posttraumatic stress disorder filtered through a clinical lens. Front Psychiatry 2019;10:368.

14. Pascual-Marqui RD. Standardized low-resolution brain electromagnetic tomography (sLORETA): technical details. Methods Find Exp Clin Pharmacol 2002;24 Supp/ D:5-12.

15. Stern Y, Neufeld MY, Kipervasser S, Zilberstein A, Fried I, Teicher M, et al. Source localization of temporal lobe epilepsy using PCA-LORETA analysis on ictal EEG recordings. J Clin Neurophysiol 2009;26:109-116.

16. Grech R, Cassar T, Muscat J, Camilleri KP, Fabri SG, Zervakis $\mathrm{M}$, et al. Review on solving the inverse problem in EEG source analysis. J Neuroeng Rehabil 2008;5:25.

17. Cowdin N, Kobayashi I, Mellman TA. Theta frequency activity during rapid eye movement (REM) sleep is greater in people with resilience versus PTSD. Exp Brain Res 2014;232: 1479-1485.

18. Begić D, Hotujac L, Jokić-Begić N. Electroencephalographic comparison of veterans with combat-related post-traumatic stress disorder and healthy subjects. Int I Psychophysiol 2001; 40:167-172.

19. Shankman SA, Silverstein SM, Williams LM, Hopkinson PJ, Kemp AH, Felmingham KL, et al. Resting electroencephalogram asymmetry and posttraumatic stress disorder. J Trauma Stress 2008;21:190-198.

20. Todder D, Levine J, Abujumah A, Mater M, Cohen H, Kaplan Z. The quantitative electroencephalogram and the low-resolution electrical tomographic analysis in posttraumatic stress disorder. Clin EEG Neurosci 2012;43:48-53.

21. Klem $\mathrm{GH}$, Lüders $\mathrm{HO}$, Jasper $\mathrm{HH}$, Elger $\mathrm{C}$. The ten-twenty electrode system of the International Federation. The International Federation of Clinical Neurophysiology. Electroencephalogr Clin Neurophysiol Supp/ 1999;52:3-6.

22. Ghumare EG, Schrooten M, Vandenberghe R, Dupont P. $A$ time-varying connectivity analysis from distributed EEG sources: a simulation study. Brain Topogr 2018;31:721-737.

23. Nichols TE, Holmes AP. Nonparametric permutation tests for functional neuroimaging: a primer with examples. Hum Brain Mapp 2002;15:1-25.

24. Breslau N. Epidemiologic studies of trauma, posttraumatic stress disorder, and other psychiatric disorders. Can J Psychiatry 2002;47:923-929.

25. Kroenke K, Spitzer RL, Williams JB. The PHQ-9: validity of a brief depression severity measure. J Gen Intern Med 2001;16:606-613. 
26. Smith BW, Dalen J, Wiggins K, Tooley E, Christopher P, Bernard J. The brief resilience scale: assessing the ability to bounce back. Int J Behav Med 2008;15:194-200.

27. Shura RD, Hurley RA, Taber KH. Insular cortex: structural and functional neuroanatomy. I Neuropsychiatry Clin Neurosci 2014;26:276-282.

28. Nagai M, Kishi K, Kato S. Insular cortex and neuropsychiatric disorders: a review of recent literature. Eur Psychiatry 2007; 22:387-394.

29. Augustine JR. The insular lobe in primates including humans. Neurol Res 1985;7:2-10.

30. Augustine JR. Circuitry and functional aspects of the insular lobe in primates including humans. Brain Res Brain Res Rev 1996;22:229-244.

31. Varjačić A, Mantini D, Levenstein J, Slavkova ED, Demeyere $\mathrm{N}$, Gillebert CR. The role of left insula in executive set-switching: lesion evidence from an acute stroke cohort. Cortex 2018; 107:92-101.

32. Kolassa IT, Wienbruch C, Neuner F, Schauer M, Ruf M, Odenwald $\mathrm{M}$, et al. Altered oscillatory brain dynamics after repeated traumatic stress. BMC Psychiatry 2007;7:56.

33. Corbo V, Clément MH, Armony JL, Pruessner JC, Brunet A. Size versus shape differences: contrasting voxel-based and volumetric analyses of the anterior cingulate cortex in individuals with acute posttraumatic stress disorder. Biol Psychiatry 2005; 58:119-124.

34. Chen S, Xia W, Li L, Liu J, He Z, Zhang Z, et al. Gray matter density reduction in the insula in fire survivors with posttraumatic stress disorder: a voxel-based morphometric study. Psychiatry Res 2006; 146:65-72.

35. Kasai K, Yamasue H, Gilbertson MW, Shenton ME, Rauch SL, Pitman RK. Evidence for acquired pregenual anterior cingulate gray matter loss from a twin study of combat-related posttraumatic stress disorder. Biol Psychiatry 2008;63:550-556.

36. Herringa R, Phillips M, Almeida J, Insana S, Germain A. Posttraumatic stress symptoms correlate with smaller subgenual cingulate, caudate, and insula volumes in unmedicated combat veterans. Psychiatry Res 2012;203:139-145.

37. Jeong H, Chung YA, Ma J, Kim J, Hong G, Oh JK, et al. Diverging roles of the anterior insula in trauma-exposed individuals vulnerable or resilient to posttraumatic stress disorder. Sci Rep 2019;9:15539.

38. Molina ME, Isoardi R, Prado MN, Bentolila S. Basal cerebral glucose distribution in long-term post-traumatic stress disorder. World J Biol Psychiatry 2010;11(2 Pt 2):493-501.

39. Simmons AN, Paulus MP, Thorp SR, Matthews SC, Norman SB, Stein MB. Functional activation and neural networks in women with posttraumatic stress disorder related to intimate partner violence. Biol Psychiatry 2008;64:681-690.

40. Critchley HD, Wiens S, Rotshtein P, Ohman A, Dolan RJ. Neural systems supporting interoceptive awareness. Nat Neurosci 2004;7:189-195.

41. Nardo D, Högberg G, Looi JC, Larsson S, Hällström T, Pagani
M. Gray matter density in limbic and paralimbic cortices is associated with trauma load and EMDR outcome in PTSD patients. J Psychiatr Res 2010;44:477-485.

42. Hopper JW, Frewen PA, van der Kolk BA, Lanius RA. Neural correlates of reexperiencing, avoidance, and dissociation in PTSD: symptom dimensions and emotion dysregulation in responses to script-driven trauma imagery. I Trauma Stress 2007;20:713-725.

43. Harricharan S, Nicholson AA, Densmore M, Théberge J, McKinnon MC, Neufeld RWJ, et al. Sensory overload and imbalance: resting-state vestibular connectivity in PTSD and its dissociative subtype. Neuropsychologia 2017;106:169-178.

44. Fonzo GA, Flagan TM, Sullivan S, Allard CB, Grimes EM, Simmons AN, et al. Neural functional and structural correlates of childhood maltreatment in women with intimate-partner violence-related posttraumatic stress disorder. Psychiatry Res 2013;211:93-103.

45. Simmons AN, Norman SB, Spadoni AD, Strigo IA. Neurosubstrates of remission following prolonged exposure therapy in veterans with posttraumatic stress disorder. Psychother Psychosom 2013;82:382-389.

46. Yin Y, Li L, Jin C, Hu X, Duan L, Eyler LT, et al. Abnormal baseline brain activity in posttraumatic stress disorder: a restingstate functional magnetic resonance imaging study. Neurosci Lett 2011;498:185-189.

47. Hlinka J, Alexakis C, Diukova A, Liddle PF, Auer DP. Slow EEG pattern predicts reduced intrinsic functional connectivity in the default mode network: an inter-subject analysis. Neuroimage 2010;53:239-246.

48. Neuner I, Arrubla J, Werner CJ, Hitz K, Boers F, Kawohl W, et al. The default mode network and EEG regional spectral power: a simultaneous FMRI-EEG study. PLoS One 2014;9:e88214.

49. Meerwijk EL, Ford JM, Weiss SJ. Resting-state EEG delta power is associated with psychological pain in adults with a history of depression. Biol Psychol 2015;105:106-114.

50. Akiki TJ, Averill CL, Wrocklage KM, Scott JC, Averill LA, Schweinsburg B, et al. Default mode network abnormalities in posttraumatic stress disorder: a novel network-restricted topology approach. Neuroimage 2018;176:489-498.

51. Hamilton JP, Farmer M, Fogelman P, Gotlib IH. Depressive rumination, the default-mode network, and the dark matter of clinical neuroscience. Biol Psychiatry 2015;78:224-230.

52. Breslau N, Davis GC, Peterson EL, Schultz L. Psychiatric sequelae of posttraumatic stress disorder in women. Arch Gen Psychiatry 1997;54:81-87.

53. Caramanica K, Brackbill RM, Liao T, Stellman SD. Comorbidity of 9/11-related PTSD and depression in the World Trade Center Health Registry 10-11 years postdisaster. J Trauma Stress 2014;27:680-688.

54. Rytwinski NK, Scur MD, Feeny NC, Youngstrom EA. The co-occurrence of major depressive disorder among individuals with posttraumatic stress disorder: a meta-analysis. I Trauma Stress 2013;26:299-309. 